\title{
Aspects of FMI in Building Simulation
}

\author{
Dipl.-Ing. Torsten Schwan ${ }^{1} \quad$ Dipl.-Ing. René Unger ${ }^{1} \quad$ B.A. Jörg Pipiorke ${ }^{2}$ \\ ${ }^{1}$ EA Systems Dresden GmbH, Germany, \{torsten. schwan, rene. unger\} @ea-energie. de \\ ${ }^{2}$ ESI ITI GmbH, Germany, Joerg. Pipiorke@esi-group. com
}

\begin{abstract}
Building physics and HVAC system simulation have become an important usage scenario of the Modelica modeling language and related simulation tools since the publication of first adequate libraries (Wetter, 2009). In 2010, the tool independent standard FMI was published in version 1.0. It enables the exchange of models between different simulation tools and even different modeling approaches. Although, automotive industry mainly initiated the FMI development, it can extensively benefit building simulation, too.

This paper describes four completely different applications of FMI in the building simulation environment which even extend the basic idea of simple model exchange. This includes the description of developed models as well as additionally required software components implementing the FMI standard.
\end{abstract}

Keywords: Building Simulation, FMI, Model-in-theloop, Controller Test

\section{Introduction}

The versatile modeling language Modelica enables engineers to model and simulate complex multiphysical problems in a wide range of different domains. Although it has been growing in the automotive industry during the late 1990s and 2000s building engineers (mainly HVAC and building physics specialists) more and more use Modelica for their purposes as well.

Since the first publications of building physics and HVAC system related Modelica libraries in 2009, a wide range of different modeling approaches have been developed. Most of them are freely available under open-source license. Some are more commercial and only partly open-source.

One of the main open-source representatives of building modeling libraries is the Modelica Buildings library of LBNL (Wetter, 2009). This constantly refined library is based on Modelica Fluid library. It focuses on detailed modeling of heating, ventilation and air conditioning systems together with detailed thermal room models. Further open-source library examples with similar modeling approaches and objectives are the Modelica Building library of RWTH Aachen (Lauster, 2012) and the Modelica
BuildingSystems library of UdK Berlin (NytschGeusen et. al., 2012). Today, these libraries as well as further similar derivatives are mainly dedicated to the worldwide growing academic community of building systems engineers and researchers.

Further commercial but partly open-source libraries like ESI ITI's Green Building (Unger et. al., 2012) and its latest derivative Green City (Schwan et. al., 2016) focus on integrated planning of sustainable and profitable solutions of buildings' and even whole cities' heat, cold and power supply.

Besides the Modelica language and derived libraries the Modelica Association has been supporting the MA Project Functional Mockup Interface (FMI) since its publication in 2010 as well. The FMI 1.0 standard was developed by 29 partners in the MODELISAR project between 2008 and 2011. In this ITEA 2 European project mainly the automotive industry forced the development of a tool independent model exchange standard. The FMI 2.0 standard followed these first developments with its publication in 2014.

The FMI standard in both versions enables engineers to exchange or co-simulate dynamic models of different domains. This way, FMI can extend the field of application of building and energy system simulation. It can furthermore help to overcome current and future limits of simulation.

Integrated planning processes more and more use accurate building physics and HVAC system models based on any kind of public or in-house library. Their main task is currently the simulation of different variants of complex building and energy system structures. This way, engineers make feasibility studies and profitableness analyzes of different system configurations. This application of building systems related Modelica libraries does not basically require FMI.

However, there is a wide range of further fields of application and benefits of Modelica in the building simulation environment. This paper describes four sample applications of FMI during integrated planning processes.

The first example supports the development of a high-level building control system including weather forecast and user prediction to optimize ecological footprint of a sophisticated multivalent HVAC system in a school and sports complex. This way, FMI 


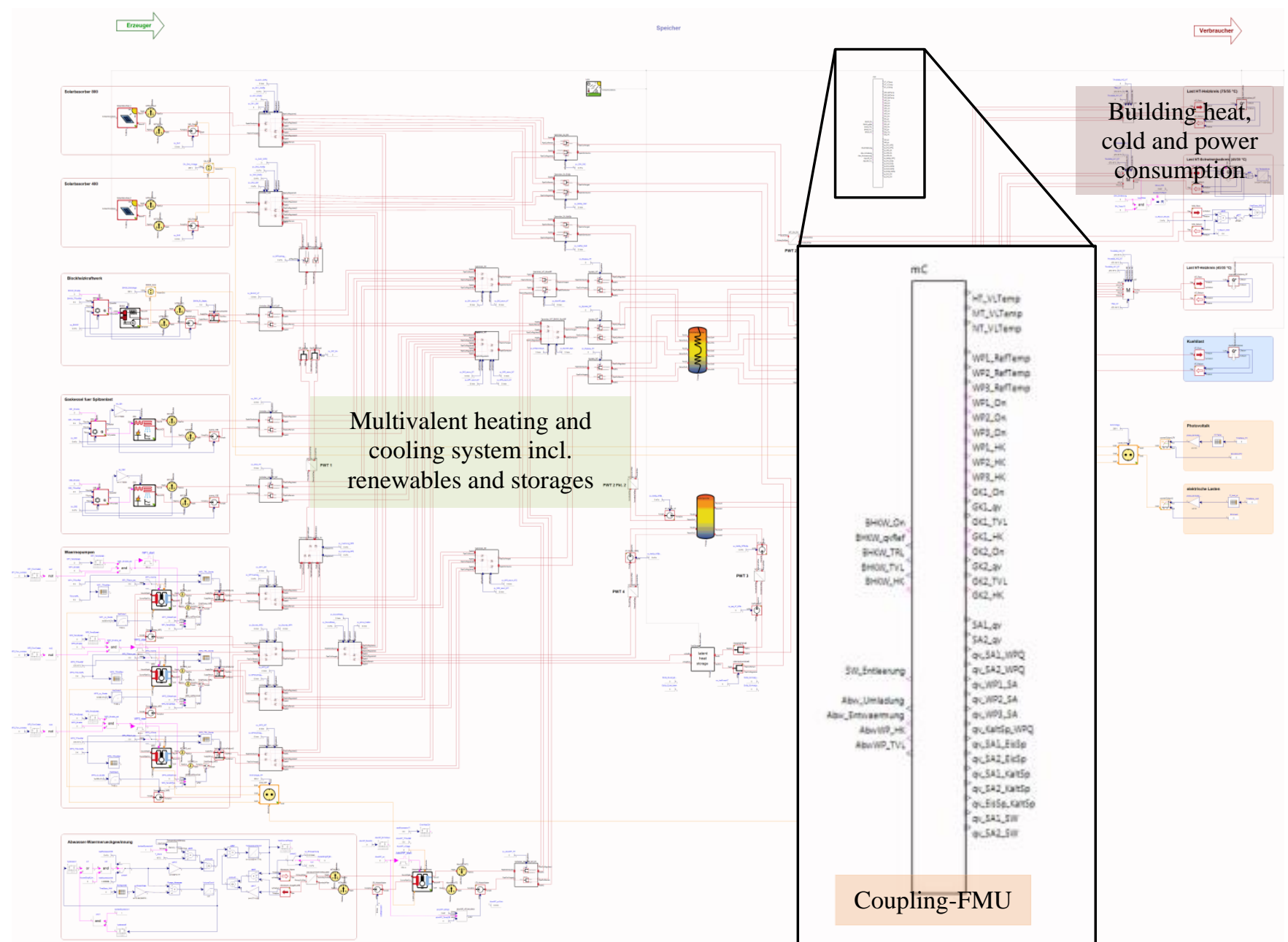

Figure 1: Modelica simulation model of the school and sports complex including coupling-FMU to external controller software (Wicke et. al., 2014)

provides the basics of a software-in-the-loop test stand of iteratively learning control software.

In the second example FMI provides the communication between a real-world HVAC system component (i.e. micro combined heat and power unit) and a complex model of the virtually connected building and hydronic heating system. This way, FMI represents the basic part of modern hardware-in-theloop test stand.

The third example uses FMI to integrate a fastcalculating simulation model in a complex virtual power plant controller. In this model-in-the-loop structure the functional mockup unit of the Modelica model helps to identify optimal operation strategies of complex diversified power plants.

The last application uses FMI to combine advantages of different simulation platforms including individually optimized numerical solvers. This way, FMI couples highly-optimized hygrothermal multizone building models with easy-to-use Modelica HVAC system models. In this case, FMI helps to separate stiff ODE systems with heavily varying time constants.

\section{Example 1 - Software-in-the-Loop}

In a small town in northern Bavaria (Germany) a local architect wants to transfer the local 1970's school and sports complex to a future 2040's energetic level. HVAC engineers therefore planned a sophisticated multivalent heat, cold and power supply system including heat pumps, cogeneration units and backup gas-fired condensing boilers as heat supply. Main heat and power source are large-scale solar thermal absorbers and photovoltaic fields. Besides an integrated waste-water heat recovery system the integration of three different storage types (stratified heat storage, cold storage and ice storage) helps to balance daily and seasonal differences between energy consumption and production (Wicke et. al., 2014).

However, this quite complex HVAC system additionally requires smart control algorithms because of the higher degree of freedom. But such iteratively learning, optimality-based control software needs sufficient testing.

The developed controller and optimization software is based on an existing Python Framework. To provide the software engineers a suitable software-in-the-loop 


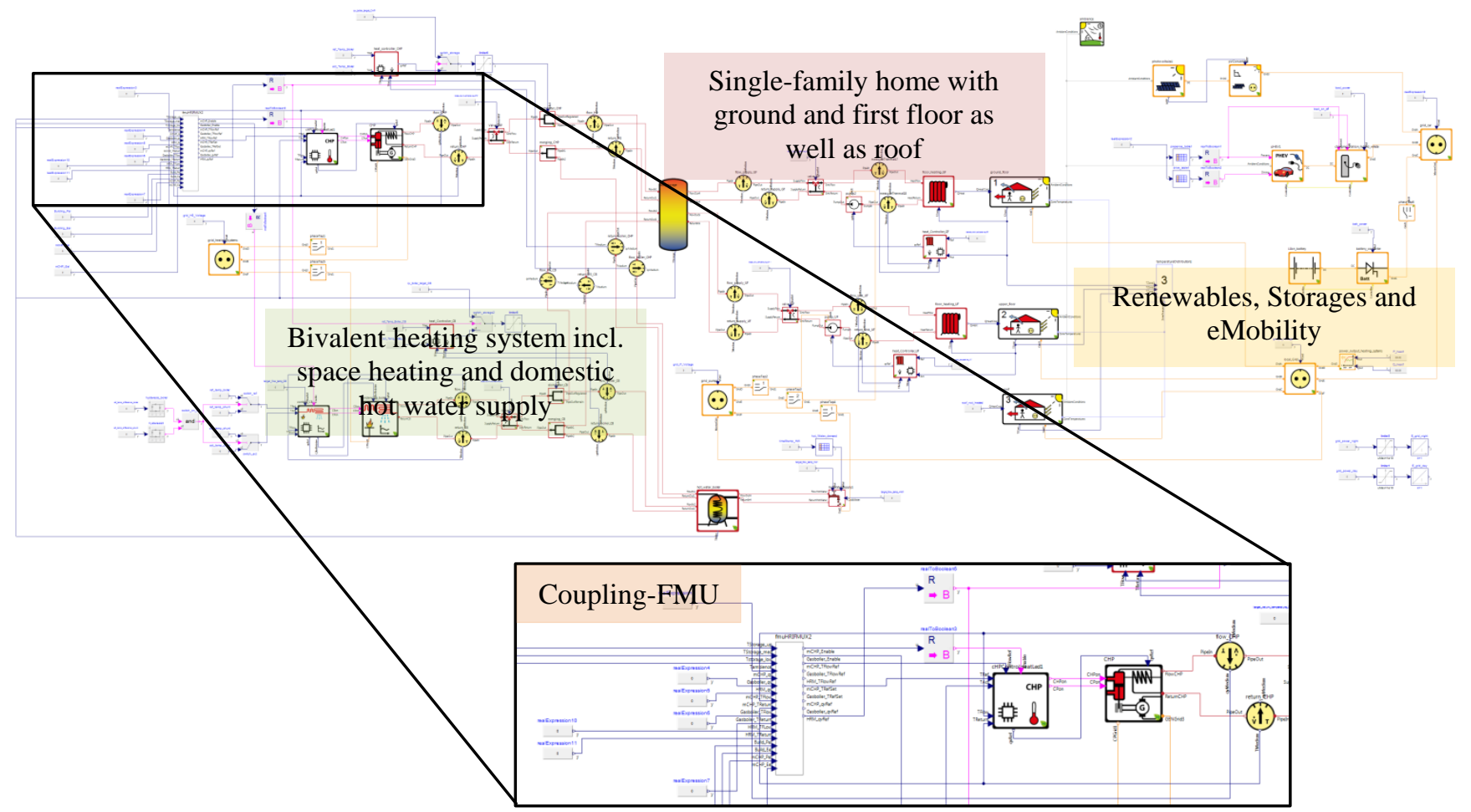

Figure 2: Modelica model the single-family-home including coupling-FMU to a real-world mCHP (Unger et. al., 2012)

test environment, a Modelica model of the developed HVAC system including the building complex's heat, cold and power demand is coupled to this Pythonbased controller software. This software-in-the-loop test bench uses the pyFMI-framework in the software and an automatically created coupling-FMU in the model (c.f. Figure 1) to establish a variable step size communication via TCP/IP protocol between model and software. This way, controller software and simulation model can run on different computer devices or even at different places.

The generation of the coupling FMU runs automatically using newly implemented FMU generator software in java. This software uses an external csv-file to define the required coupling interface (inputs and outputs of coupling FMU) between controller software and test model.

Software-in-the-loop tests of new controller software require extensive scrutinizing regarding internal controller timings, especially in combination with PI or PID controllers. The integrator time constants have great influence on later robustness. Building simulation models normally run (much) faster than real-time to provide sufficient results (at least one year) within adequate time periods. Those time constants therefore have to be adapted regarding communication time steps as well as a constantly synchronized real-time factor.

\section{Example 2 - Hardware-in-the-Loop}

Existing complex building structures, like the school and sports complex in Figure 1, require smart solutions for energetic renovations. But also smaller buildings, like simple single-family homes, require holistic optimization approaches of heat and power supply. Photovoltaic and cogeneration units this way enable massive reductions of overall annual power consumption with manageable investment costs.

Photovoltaic (PV) modules provide renewable power and small cogeneration modules (so-called micro combined heat and power units - mCHPs) utilize synergy effects of heat and power production.

To enable a wide range of different system configurations as well as an ongoing system optimization CHP and PV manufactures have to constantly improve their products. This includes controller software as well as hardware components. But reliable developments again require sufficient testing. Hardware tests of single system components can use simple test stand configurations (e.g. mCHP heat storage, controlled recooling) and generically calculated heat and power load profiles. But dynamic tests of hardware and software with evaluated realworld-conditions need further expenses.

This way, Modelica models in combination with FMI can again help to provide an easy-to-use platform for hardware-in-the-loop tests. Figure 2 shows a simple example of a developed single-family home model for a hardware-in-the-loop test of a small mCHP. This single-family home model includes heat and power consumption of a small house (3-thermal zones), renewable power production and storage by photovoltaic modules, a battery and a connected eVehicle charging infrastructure. Heat is supplied by a $2.5 \mathrm{~kW} \mathrm{mCHp}$ with $1.0 \mathrm{~kW}$ power output and a peak- 


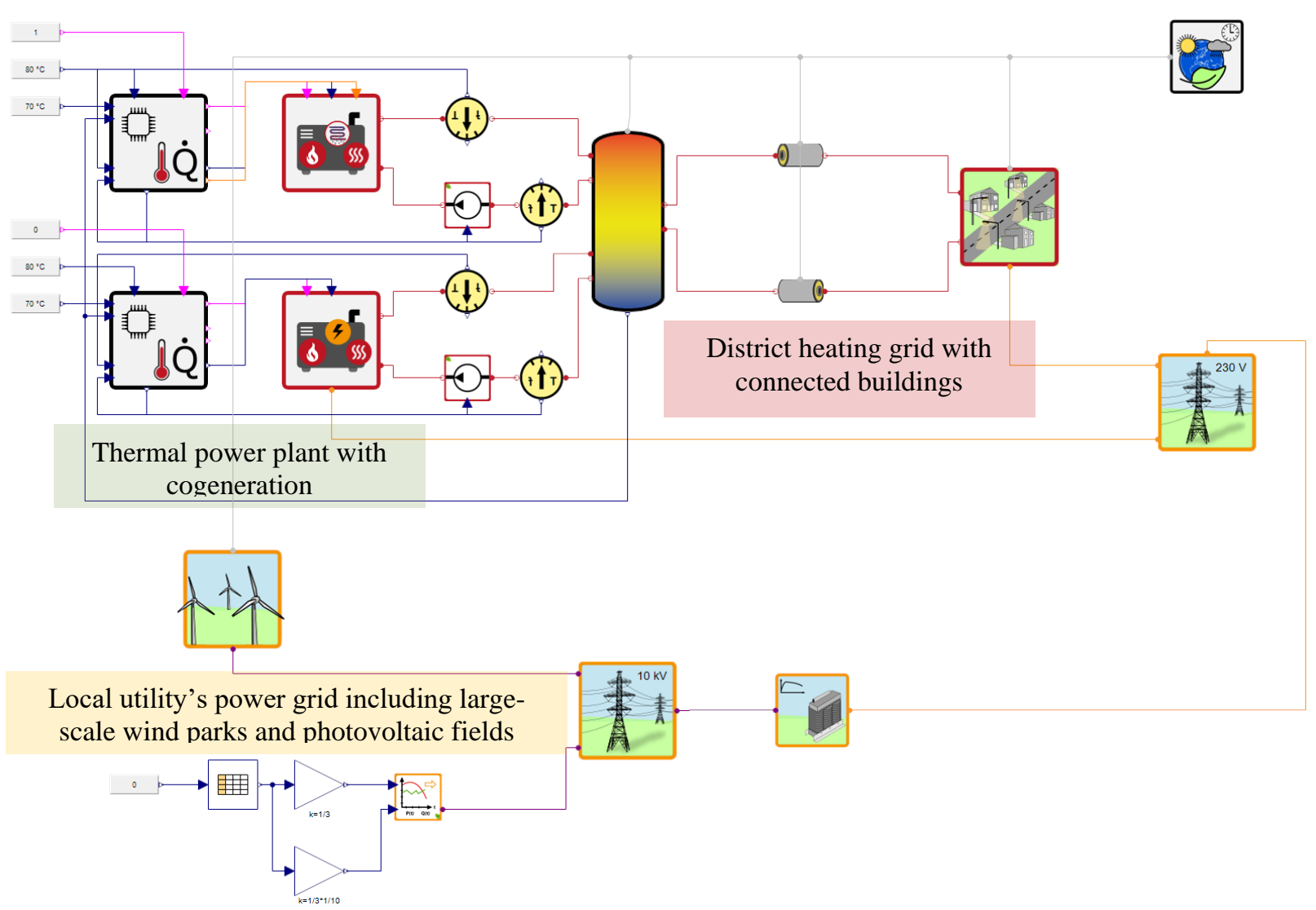

Figure 3: Modelica model of a simple district heating grid with cogeneration plant and superior electric grid connection (Schwan et. al., 2016)

power gas-fired condensing boiler (Unger et. al., 2012).

The real-world counterpart of the $\mathrm{mCHP}$ is connected to the model via a specific coupling FMU. This again automatically created FMU connects realworld measurement sensors (heat and power sensors of the $\mathrm{mCHP}$ ) with the model. Measured heat and power is added as additional heat and power supply to the building. The $\mathrm{mCHP}$ controller gets temperature measurements of the simulated building (ground and first floor) and the heat storage to start and stop the real-world mCHP engine. Furthermore, the mCHP controller decides to run the simulated peak-power boiler if reference temperatures are underrun.

The required coupling FMU (c.f. Figure 2) couples Modelica inputs and outputs in the model with realworld digital and analogue signals of the $\mathrm{mCHP}$ controller. Therefore, the FMU internally converts model variables into TCP/IP protocol compatible signals. These signals are interchanged between the simulation computer unit and the local PLC (Programmable Logic Controller) of the hardware-inthe-loop test stand. This PLC directly communicates with the mCHP controller via a system specific communication bus (e.g. Modbus). Heat supply to recooler and power supply to test stand's grid are measured with electronic sensors which send back the measurement data to the model.
This configuration represents a simple closed-loop hardware-in-the-loop test stand based on Modelica models and FMI. Again timing balance between realworld-time and simulation time is highly important. In opposite to the software-in-the-loop approach a hardware-in-the-loop test stand cannot easily be accelerated. It requires rigid real-time synchronization between model and device under test. This avoids the adaption of controller-internal timing constants.

Fortunately, building physics and HVAC system models mostly are (much) faster-than-real-time. Model acceleration is not necessary. The model even has to be stopped after each communication step to synchronize simulation time and real-world time.

\section{Example 3 - Model-in-the-Loop}

Modelica models as well as the Functional Mockup Interface standard will be a major part of future sophisticated test stands (both hardware- and softwarein-the-loop) in the building sector. However, both provide more potential to improve the current situation of planners and engineers in this environment.

FMI was developed to provide an independent model exchange and co-simulation standard. Besides using FMI as a pseudo data exchange interface, Modelica models of sophisticated building and HVAC system structures can also be exported as standalone FMUs. 


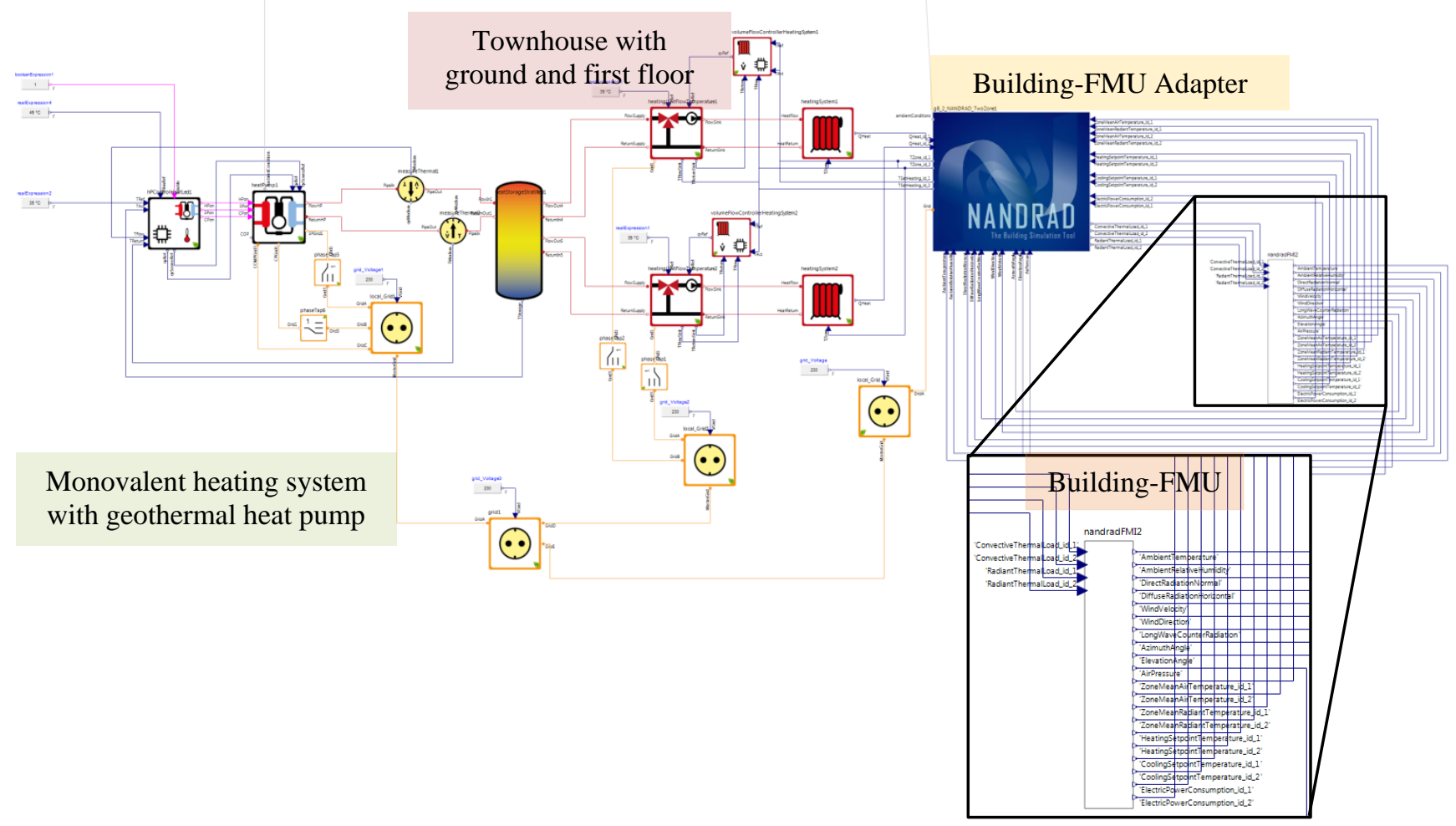

Figure 4: Coupling of a Modelica HVAC system model (SimulationX/Green City) with a TU Dresden building-FMU based on the building physics simulation tool Nandrad (Paepcke et. al., 2016)

The integration of these FMUs in upcoming modelin-the loop controller architectures (e.g. Virtual Power Plant Controller - c.f. Schwan et. al., 2016) helps building engineers to design optimality-based controllers which can increase overall system efficiency without major extra investment costs.

Figure 3 shows such a simple Modelica model based on ESI ITI's Green City library. It represents a highlysimplified model of a cold district heating grid with decentral heat pumps, a thermal power plant with cogeneration and the local utility's power grid including wind parks and photovoltaic fields. This system is a role-model of a future part of a virtual power plant which provides balancing power to the transmission grids via energy exchange (EEX).

Available heat storage capacities in the district the heating grid provide virtual storage capacities for renewable power surplus of wind parks or photovoltaic (i.e. negative balancing power). Furthermore, decentral heat pumps can partly run as renewable energy dump as well by utilizing each building's thermal capacity. In times of grid deficits, the district heating grid reduces the power consumption of the heat pump and the cogeneration plant provides positive balancing energy.

These strategies will help to decarbonize overall energy supply of buildings in the future. However, such concepts require major investments and refinancing is not possible with diminishing energy profits. But the sale of balancing energy at the energy exchange (c.f. EEX) will help to partly compensate resulting add-on costs. This requires though highlyaccurate prediction of available storage capacities.

The integration of accurate but fast calculating simulation models of those grids in energy trading tool chains and corresponding system controllers (e.g. controller of a cogeneration plant) increases profit reliability at the balancing energy exchange. Therefore, these models are exported including a suitable numerical solver as independent FMUs.

Then, these FMUs are simulated for comparatively short time periods (i.e. few days ahead) to identify maximum profit margins regarding different upcoming weather conditions as well as expected power and heat consumption profiles.

In this model-in-the-loop configuration the models must run much-faster-than-real-time to enable the evaluation of a great number of different input parameter sets and influencing characteristics. Synchronization is not needed because the controller only uses the accumulated simulation results.

\section{Example 4 - Co-Simulation}

All three previously shown FMI applications in the building sector mainly use the standard to provide sophisticated test scenarios or to optimize controller functionality. However, FMI is also applicable in this environment in its inherent manner, a model exchange and co-simulation standard.

Complex building models combine a vast number of different physical components with highly diversified 
time constants. Building physics mainly represent slow processes. Heating up or cooling down walls or whole buildings need several hours to several days depending on thermal inertia and available heating/cooling system configurations. Power supply of renewables, e.g. photovoltaic modules or wind power plants, can fluctuate within several seconds or few minutes depending on cloudiness and surrounding shadings. Inverter controllers of photovoltaic, batteries or even eMobility charging stations can react within a few seconds regarding available measurements (e.g. inhabitants' individual power consumption in a dwelling house).

This great variety of time constants can cause very stiff ODE systems in one Modelica model. But FMI enables to separate models regarding different time constants or to combine the strength of different simulation tools and solvers. Coupled models can cosimulate specialized building physics models with highly optimized PDE solvers (e.g. heat and moisture transport through walls) together with fast and accurate HVAC system models in Modelica and its therefore optimized ODE solvers.

Figure 4 therefore shows the coupling between Modelica HVAC system models based on ESI ITI's Green City library coupled to a townhouse building physics model in TU Dresden's Nandrad simulation environment (Paepcke et. al, 2016). This way, a Modelica simulation environment imports the Building-FMU created in Nandrad as an additional model component. The townhouse model consists of two thermal zones representing ground and first floor. The HVAC system includes a monovalent heat pump and a heat storage which provides the heat to the two hydronic heating circuits.

However, both FMI 1.0 and FMI 2.0 only allow scalar interface variables (inputs and outputs). But especially complex building physics models require a high number of common interface variables (e.g. indoor temperatures and temperature set points of all thermal zones or rooms). Therefore, Nandrad additionally provides a building-FMU adapter which automatically connects the bus interfaces in the Modelica model with the vast number of scalar inputs and outputs of the building-FMU.

On the one hand, this approach provides an interface between a Modelica HVAC system model and an imported building physics model. Co-simulation this way uses basic master algorithms of the available Modelica simulation environments, like ESI ITI's SimulationX. On the other hand, the HVAC system model including the required adapter can be exported as standalone FMU, too. This way, co-simulation between two or more FMUs can use more specialized master algorithms (Clauß et. al., 2016) to optimize simulation speed and accuracy. This can furthermore improve the tradeoff between increase of simulation speed by usage of several individually optimized solvers and speed reduction by adding additional communication time between the FMUs.

\section{Conclusion}

This paper presents an overview of different fields of application of Functional Mockup Interface standard in the building simulation environment. Building and HVAC system simulations currently become one major domain of the Modelica language including an increasing number of available academic and commercial libraries.

The Modelica community also focuses on coupling of different models as well as software and hardware components to utilize synergy effects and to extend Modelica's fields of application (e.g. BCVTB Nouidui et. al., 2014). Because of its tool independency, its industrial support and its adjustability to latest network and internet technologies, the FMI standard and its upcoming updates will help to integrate Modelica in all design, test and validation processes in the building sector within the next years.

\section{References}

M. Wetter. A Modelica-based model library for building energy and control systems, $11^{\text {th }}$ International IBPSA Conference, Glasgow, 2009.

M. Lauster. Modelica Building Library and Building Models. Symposium on Integrated Planning and Simulation in Building Physics and Technology. Dresden, 2012.

C. Nytsch-Geusen, J. Huber, M. Ljubijunkic, J. Rädler. Modelica-BuildingSystems - A Simulation Library of complex Building Energy Systems. BauSIM, Berlin, 2012.

R. Unger, T. Schwan, B. Mikoleit, B. Bäker, C. Kehrer, T. Rodemann. "Green Building" - Modelling renewable building energy systems and electric mobility concepts using Modelica. $9^{\text {th }}$ International Modelica Conference, Munich, 2012.

T. Schwan, R. Unger. Holistic District Heating Grid Design with SimulationX / Green City. ESI SimulationX User Forum, Dresden, 2016.

M. Wicke, T. Schwan, R. Unger. Model-based design of control strategies for a sophisticated building energy system in a school and sports complex. $17^{\text {th }}$ ITI Symposium, Dresden, 2014.

A. Paepcke, A. Nicolai, T. Schwan. Interfaces of CoSimulation Coupling between Building and Heating System Simulation. Central European Symposium on Building Physics, Dresden, 2016.

C. Clauß, K. Majetta, R. Meyer. Development of Simulator Coupling Algorithms using FMI Interface for Building Simulation Applications. Central European Symposium on Building Physics, Dresden, 2016.

T. S. Nouidui, M. Wetter. Tool coupling for the design and operation of building energy and control systems based on the Functional Mock-up Interface standard. $10^{\text {th }}$ International Modelica Conference, Lund, 2014. 